The Council has approved the election of the following candidates to the grade of Full Member:

\begin{tabular}{|c|c|c|c|}
\hline Mika Aaltonen & John E. Brown & Susan C. Dunne & Jonathan $\mathrm{H}$. Jiang \\
\hline Amanda S. Adams & George H. Bryan & Craig C. Epifanio & Xun Jiang \\
\hline Rashid A. Akmaev & Mark Buehner & Benjamin M. Erskine & Therese M. Jorgensen \\
\hline Jose J. Alamo & Robert J. Burgman & Amanda G. Evans & Andrew Just \\
\hline Jason R. Anderson & Jessica Callahan & Riccardo Farneti & Erik D. Kabela \\
\hline Sam Argier & Megan B. Campbell & Paul Fitzsimmons & Ralph A. Kahn \\
\hline Robert L. Ashbaugh & Teresa Canavan & Amy L. Flanagan & Masahiro Kazumori \\
\hline Richard T. Austin & Christopher P. Cappella & Steven R. Fleegel & Matthew J. Keefe \\
\hline Svetlana M. Bachmann & James M. Caron & Akeem A. Folajin & Gerald J. Keeler \\
\hline Andrew Barkwith & Keith Cavey & Filipe D. Freires-Lucio & Ronald H. Kellerman \\
\hline Janet F. Barlow & Joseph J. Charney Jr. & Richard B. Friesen & Kristin E. Kline \\
\hline Mary C. Barth & Fang-Yi Cheng & Justin M. Fujioka & Jaclyn K. Kost \\
\hline Paul J. Bayer & Keith A. Cherkauer & Janet E. Gahagan & Andrew Kozak \\
\hline Rowland J. Beardsell & Mary Ann Chory & Bradwell J. Garanganga & James P. Kramper \\
\hline Scott M. Beaver & Kristin M. Clark & Derrill T. Goldizen & Thomas Kuba \\
\hline Michael G. Bedard & Peter U. Clark & Eric J. Gose & Mary Ann Kutny \\
\hline Kristopher M. Bedka & Justin Cooke & Jason Grzywacz & Douglas M. Landwehr \\
\hline Kyle S. Berg & Lacey M. Coolidge & Haoyu Gu & Ruth A. Lane \\
\hline Sachin B. Bhardwaj & Wilton L. Cooper & Zhichang Guo & Stephen P. Leatherman \\
\hline Eric Bickel & Gary K. Corlett & Rebecca Haacker-Santos & Thierry Leblanc \\
\hline Geoff Bing & Lee M. Cronce & Tapio Haarlaa & Kyle S. Lerman \\
\hline Ernie Blair & Thomas M. Cronin & Tomas Halenka & Bailing Li \\
\hline John A. Bognar & Geoffrey Crowley & Todd Halter & Brad Lindseth \\
\hline John Bolten & Dacian N. Daescu & Bjarne K. Hansen & Elita Loresca \\
\hline Sandrine Bony & Spencer T. Daines & John Harlim & Sarah E. Love \\
\hline Steven G. Bowen & Luis Gustavo G. de Goncalves & Mark Hendrickson & JianhuaLu \\
\hline Michael E. Bowler & Ruben Delgado & Redina L. Herman & Janet K. Lynch \\
\hline Zach Brandman & Julie L. Demuth & Michael Hesse & Clinton P. MacDonald \\
\hline Kyle Brehe & Kathleen Dibling & Michael Holland & Donald F. Mack Jr. \\
\hline Paul Bridge & David S. Dimitriou & Harrison C. Hove & Theresa S. Martin \\
\hline Sarah D. Brooks & Catia M. Domingues & James R. Howard & Deborah J. Martorell \\
\hline
\end{tabular}

The Council has approved the election of the following candidate to the grade of Full Member with Student

\title{
Privileges:
}

\author{
Nicholas R. Allard \\ Stephanie J. Armstrong \\ Carl P. Densford \\ Michael W. Egner \\ Gina M. Eosco \\ Thioro Fall \\ G. Gutierrez Garcia
}

\author{
Alex Harrington \\ Mohamed M. Hefny \\ Barbara M. Johnston \\ Marina L. Jurica \\ Steven V. Merkel \\ Akhilesh K. Mishra \\ Richard H. Moore
}

\author{
Hyacinth C. Nnamchi \\ Swagata Payra \\ Heikki Pohjola \\ Wenjun Qian \\ David R. Roache \\ Ahira M. Sanchez-Lugo \\ Wilfrid Schroeder
}

\author{
Jeffrey R. Taylor \\ Barbara Tencer \\ Adam B. Thomas \\ Douglas Tobias \\ Amber E. Wakefield \\ Meng Zhang
}

The Executive Committee has approved the election of the following candidates to the grade of Associate Memberl

\section{K-12 Teacher:}


Andreas Marx

William G. Massey

Ryan M. Matthews

Daniel P. Matusiewicz

Rob Mayeda

Harry E. Mayer

Sarah L. McKenna

Thomas J. McNellis Jr.

Jean L. Michael

Gonzalo Miguez-Macho

David A. Miller

Zinedeme Minia

Gary A. Morris

Christopher R. Morrow

John P. Morse

Matthew D. Mosbacher

Qiaozhen $\mathrm{Mu}$

John Murray

William Murtagh

Michael S. Mussetto

Bradley C. Navarro

Jeffrey L. Nelson

Janice Newman

Bruce J. Nicholson

Robert L. Norton

Thomas P. O'Brien III

Heather A. O'Malley

Laurie A. O'Reilly

Eduardo P. Olaguer Jr.

Michael C. Ortega

Corwin M. Parker
Joseph C. Pasquarelli

Stacey Pensgen

John C. Petheram

Alexis Phillips

Yelena L. Pichugina

Ana C. T. Pinheiro

Arastoo Pour Biazar

Dylan C. Powell

Albert J. Ramon

Alan Ramyond

Leslie Remme

Jeff S. Robbins

Robert E. Robins

Wendy A. Ryan

Mark A. Sannutti

Tomonori Sato

Gavin A. Schmidt

Andrea B. Schumacher

Matthew P. Seidel

Eddie L. Sheerr

Li Shi

Merit M. Shoucri

Richard J. Siltzer

Kristen N. Slocum

Eric R. Snodgrass

Lee S. Solomon

Candice L. Sorensen

Phillip L. Spencer

Kelly Sponberg

Kyle Spyksma

Neptune Srimal
O. C. St. Cyr

James Stagliano

Cristiana Stan

Gheorghe Stancalie

Matthew R. Steinbugl

Clayton B. Stiver

Eric J. Stone

Joshua M. Stone

Shenna Storr

Jochen Stutz

Jai Sukhatme

William Sullivan

Hilding Sundquist

Geri Swanson

Edward H. Teets Jr.

Shahram Tehranian

Brian R. Teigland

Mark A. Tew

Christoph K. Thomas

Stephen J. Thomas

Victor Thomas

Gregory D. Thompson

Kimberly A. Thomson

Anthony J. Tomaro

Marc Touchton

Yu-heng Tseng

Dana M. Turtle

James B. Ussery

Michael P. Utley

Petrus Van Oevelen

William D. Vanderbrink
Fritz VanWijngaarden

Arthur P. Verbin

Lucie A. Vincent

Danielle Vollmar

James C. Walter

Matt Wanink

David A. Warde Sr.

Jeff Weber

Michael Wehner

Paul A. Wetzl

Derek L. Williams

Patrick C. Williams

Samuel P. Williamson

Michael H. Wooster

Man-Li C. Wu

Yonghui Wu

Yu-ling Wu

Teddy J. Wykle Jr.

Jenifer L. Xanders

Hongjie Xie

Xiaobiao Xu

Huiwen Xue

Eun-Su Yang

Kunio Yoneyama

Nedjeljka Zagar

Chao-lin Zhang

Guifu Zhang

Yong Zhang

Daniel K. Zhou

Ping Zhu

The Executive Committee has approved the election of the following candidates to the grade of Associate Member:
Paul Bagdade
Ginger L. Brookover
Milenko Cilic
Eddy Crick

\author{
Henry DeBianchi \\ Roberta M. Gonzales \\ Fiona Gorostiza \\ Bob Josuweit
}

\author{
Michael D. Keebaugh \\ Meredith L. Leonard \\ Jeffrey A. Logan
}

Steve Mitchell

Betty H. Morrow

Gene Rosendahl

The Executive Committee has approved the election of the following candidates to the grade of Associate

\section{Member-Precollege Student:}

Victor Alari

Kyle V. Collett

Khalil Cowart

Adam C. Denny
Elizabeth A. DiGangi

Sean Ernst

Nicholas R. Esposito
Caelin B. Kelsey

David S. Megginson

Jason D. New
Chelsea D. Patton

Morgan R. Sills

Thomas Stavola Jr. 
The Executive Committee has approved the election of the following candidates to the grade of Student Member:

Melissa Ackerman
Mary G. Adkins
Rachel I. Albrecht
Joel K. Alderfer
Troy R. Allison
Dan K. Arthur
Jonas V. Asuma
Brenda L. Babin
Daniela Lopez Barragan
Gregory Battle
Brad Beechler
Kristi Bernstein
Alexis A. Bloom
Alamelu Brooks
Peter M. Caldwell
Karina A. Castillo
Anne P. Chalmers
Marcelo Chamecki
Ching-Yee Chang
Lung-Yao Chang
Erica Clay
Angela J. Colbert
Tina L. Colbert
Savannah A. Collins
Thomas W. Collow
Douglas Crauder
Jeffrey Henry Curtis
Gregory J. Cutrell
Channing R. Dale
Melissa D. Dawson
Robert L. Deal
Sam S. Dienst
Cheikh Abdoulahat Diop
Ken P. Dixon
James B. Duncan Jr.
Michael J. Dvorak
Crystal J. Egger
Caitlin L. Faerevaag
Alejandro N. Flores
Abigail B. Fontaine
Bonne J. Ford
Evelyn R. Frazier
David J. Gagne II
Chandana Gangodagamage Sr.
Brett Gantt
Chaim I. Garfinkel
Guoqing Ge

Autumn D. Glasgow

Jonathan Gomez

Sergio I. Gonzalez Sr.

Kristen L. Gore

Uriah M. Gravois

Shelly Guare

Moussa Gueye

Eric M. Guillot

Brian A. Haines

David Harker

Alexander E. Hasha

Michael G. Haynes

Jill N. Henriksen

Nathan C. Hiers

Jordan S. Higley

Erica Hill

Heather D. Hollingsworth

Kelli L. Hornberger

Katherine L. Howard

Christopher D. Hoyt

Rachel Hughes

Karla Y. Hyde

Ashley A. Jones

Lauren J. Jones

McArthur Jones Jr.

Linda Jordan

Hsun-Ying Kao

Yohai Kaspi

David P. Keeney

Josh Keller

Chang Ki Kim

Seung Hee Kim

Allison J. King

Walter C. Kolczynski Jr.

William A. Komaromi

Leila K. Konkola

Laura Kruep

Jonathan T. Kurtz

Joseph B. Land

Sally L. Lavender

Lauren A. Lavo

Rhonda A. Lee

Justin B. Leo

Anita LeRoy

Jessica M. Liptak

Sergey Livshits
Jeremy Lorber

Bryan T. Losier

Katherine S. Maclay

Eric J. Mailloux II

Tamil Maldonado-Vega

Jessica A. Markle

AllisonMarquardt

Joss Matthewman

Mamadou L. Mbaye

Jennifer L. McCaskill

Rachel R. McCrary

Luke McGuire

Meghan K. Mee

Ryan M. Meyer

Yumin Moon

Peter J. Moore

Heather N. Morgan

Scott Mowaswes

Robbie M. Munroe

Matthew S. Munsey

Edward J. Natenberg

Emily L. Niebuhr

Thomas A. Noble

Alexandros A. Ntelekos

Heather L. O'Neill

Brett G. Odom

Hoon-Ju Oh

Michael Oliver

Alexis O. Orengo

Raquel Orozco

Ying Pan

Clark D. Payne

Travis W. Phipps

Sara E. Presnall

Heather Quantz

Destiny C. Rainney

Ismari Ramos Medina

Nikki-Dee Ray

Joseph G. Reedy

Rachel G. Rogers-Van Nice

Miguel O. Roman III

Kevin Romero

Jill C. Ruddon

Seyni Salack

Aaron K. Sanders

Thomas B. Sawyer
Christopher J. Schaffer

Victoria R. Schemelia

Megan C. Schwitzer

Rony Marcel Semexant

Victoria L. Shaw

Xiaogang Shi

Shraddhanand Shukla

Courtney M. Siegert

Victoria A. Sinclair

Daya E. Snapp

Guan Song

Julie A. Soper

Kyle J. Spangle

Chris Spannagle

Audra Spencer-Goldstick

Michael P. St. Laurent Jr.

Jutta C. Stark

Cassie A. Stearns

Levi K. Stewart

David A. Sutherland

Breanne J. Templeton

Daniel Thompson

Brentha Thurairajah

Jorge A. Torres

Michael D. Toy

Vu Tran

John C. Turner

Christopher Valentine

Kathryn L. Van Werkhoven

Carlos A. Velasco

Stephanie N. Waterman

Jason R. Webster

Julia L. Weiden

Melissa A. Weiss

Graham S. Weston

Dustin E. Williams

Jeff Willison

Christopher J. Wilson

Mattias Winterdahl

Amanda L. Wisdom

Colin M. Zarzycki

Yan Zhang

Chunhua Zhou

Li Zhou

Yan Zhou

Maggie R. K. Zimmerman 